\title{
Examining the possibility of an acquired deficit in audiovisual temporal perception for speech and musical events Argiro Vatakis
}

\author{
Address: Crossmodal Research Laboratory, Department of Experimental Psychology, University of Oxford, Oxford, UK \\ from International Society on Brain and Behaviour: 3rd International Congress on Brain and Behaviour \\ Thessaloniki, Greece. 28 November - 2 December 2007 \\ Published: 17 April 2008 \\ Annals of General Psychiatry 2008, 7(Suppl I):SI37 doi:I0.II86/I744-859X-7-SI-SI 37
}

This abstract is available from: http://www.annals-general-psychiatry.com/content/7/SI/SI37

(C) 2008 Vatakis; licensee BioMed Central Ltd.

\section{Background}

Multisensory interactions between audiovisual-speech inputs have primarily been investigated in healthy participants with only a limited number of studies identifying patients with a disruption of audiovisual perception as a consequence of other neurological deficits. A specific disruption of audiovisual speech integration has, in fact, never been reported until recently by [1]. They described patient AWF who experienced a temporal mismatch in audiovisual speech in the absence of any language/sensory impairment. Similarly, patient RW reported perceiving auditory-speech as occurring earlier in time than the corresponding visual-speech. Initial testing revealed that RW indeed appeared to have a problematic temporal percept of audiovisual speech. Methodological problems, however, led to the inconclusiveness of these results.

\section{Materials and methods}

In the present experiments, video clips with auditory/visual delays of $\pm 300, \pm 200, \pm 133, \pm 66$, and 0ms were presented to RW. RW had to decide on each trial whether the auditory- or visual-signal appeared to have been presented first. The video clips consisted of: a female BritishEnglish speaker uttering/aba/ and /aga/ and the bird's-eye views of a female's fingers playing the piano notes ' $\mathrm{c}$ ' and ' $f$ '. All video clips were $800 \mathrm{~ms}$ long.

\section{Results}

Analysis of RW's data revealed the absence of any impairment in the temporal perception for either speech or nonspeech events. Specifically, RW's sensitivity to the asynchrony present in speech and music was $67 \mathrm{~ms}$ and $70 \mathrm{~ms}$, respectively. These values are similar to those previously obtained for healthy participants [2]. Comparison of RW's data with controls revealed no differences $[\mathrm{F}<1$, n.s.], with control participants' sensitivity to asynchrony in speech and music being equal to $66 \mathrm{~ms}$ and $76 \mathrm{~ms}$, respectively.

\section{Conclusions}

The methodological problems of previous testing could have led to RW being misdiagnosed. However, well-controlled experimentation revealed that RW's temporal percept was within the temporal limits of normal participants.

\section{Acknowledgements}

I would like to thank Dr. Foxton and Dr. Spence for their help. A. V. was supported by a Newton Abraham Studentship from the Medical Sciences Division, University of Oxford.

\section{References}

I. Hamilton R. H., Shenton J. T., Branch-Coslett H.: An acquired deficit of audiovisual speech processing. Brain \& Language 2006, 98:66-73.

2. Vatakis A., Spence C.: Audiovisual synchrony perception for music, speech, and object actions. Brain Research 2006, IIII:134-I42. 